\title{
Gold Nanoparticles interacting with Synthetic Lipid Rafts: an AFM investigation
}

\section{Authors}

Andrea Ridolfi* †,1,2,3, Lucrezia Caselli ${ }^{\dagger, 3}$, Costanza Montis ${ }^{1,3}$, Gaetano Mangiapia4, Debora Berti $^{1,3}$, Marco Brucale ${ }^{1,2}$ and Francesco Valle ${ }^{1,2}$

\section{Affiliations}

${ }^{1}$ Consorzio Interuniversitario per lo Sviluppo dei Sistemi a Grande Interfase (CSGI), via della Lastruccia 3, 50019 Florence (Italy).

${ }^{2}$ Consiglio Nazionale delle Ricerche, Istituto per lo Studio dei Materiali Nanostrutturati (CNRISMN), via Gobetti 101, 40129 Bologna (Italy).

${ }^{3}$ Dipartimento di Chimica "Ugo Schiff", Università degli Studi di Firenze, via della Lastruccia 3, 50019 Florence (Italy).

${ }^{4}$ GEMS am Heinz Maier-Leibnitz Zentrum (MLZ). Helmholtz-Zentrum Geesthacht GmbH, Lichtenbergstr. 1, D-85747 Garching, Germany.

${ }^{\dagger}$ A.R. and L.C. contributed equally to this work.

*Corresponding author. Email: andrea.ridolfi@ismn.cnr.it

\section{Abstract}

Inorganic nanoparticles (NPs) represent promising examples of engineered nanomaterials, providing interesting biomedical solutions in several fields, like therapeutics and diagnostics. Despite the extensive number of investigations motivated by their remarkable potential for nanomedicinal applications, the interactions of NPs with biological interfaces are still poorly understood. The effect of NPs on living organisms is mediated by biological barriers, such as the cell plasma membrane, whose lateral heterogeneity is thought to play a prominent 
role in NPs adsorption and uptake pathways. In particular, biological membranes feature the presence of rafts, i.e. segregated lipid micro and/or nano-domains in the so-called liquid ordered phase $\left(L_{\circ}\right)$, immiscible with the surrounding liquid disordered phase $\left(L_{d}\right)$. Rafts are involved in various biological functions and act as sites for the selective adsorption of materials on the membrane. Indeed, the thickness mismatch present along their boundaries generates energetically favorable conditions for the adsorption of NPs. Despite its clear implications in NPs internalization processes and cytotoxicity, a direct proof of the selective adsorption of NPs along the rafts' boundaries is still missing to date. Here we use multicomponent Supported Lipid Bilayers (SLBs) as reliable synthetic models, reproducing the nanometric lateral heterogeneity of cell membranes. After being characterized by Atomic Force Microscopy (AFM) and Neutron Reflectivity (NR), multi-domain SLBs are challenged by prototypical inorganic nanoparticles, i.e. citrated gold nanoparticles (AuNPs), under simplified and highly controlled conditions. By exploiting AFM, we demonstrate that AuNPs preferentially target lipid phase boundaries as adsorption sites. The herein reported study consolidates and extends the fundamental knowledge on NPs-membrane interactions, which constitute a key aspect to consider when designing NPs-related biomedical applications.

\section{INTRODUCTION}

Despite the impressive technological advancement in the design of "smart" inorganic nanoparticles (NPs), their impact on biological systems and related toxicity are still poorly understood $^{1,2}$, limiting their effective clinical translation. The interaction of engineered nanomaterials, either intentionally or inadvertently released into the environment, with living organisms is mediated by biological barriers, such as cell plasma membranes, which primarily determine NPs biological fate and cytotoxicity ${ }^{3}$. Therefore, the interaction of NPs 
with biological interfaces is a key research topic, aiming at the safe use of nanotechnology and maximization of its potential in therapeutics and diagnostics ${ }^{4,5}$.

In this framework, lipid-based synthetic model membranes are useful platforms to mimic biological interfaces under simplified conditions, allowing for the identification of key determinants regulating nano-bio interactions ${ }^{6-8}$. Supported Lipid Bilayers (SLBs) are often used as $2 \mathrm{D}$ biomembrane models ${ }^{9,10}$, enabling to precisely tune their physicochemical properties and avoiding the complications related to the 3D nature of biological membranes. They also represent versatile and promising platforms for the development of biosensors ${ }^{11}$ and technological assays for biological applications ${ }^{12}$.

In addition, multicomponent SLBs models allow studying the lateral compositional heterogeneity that characterizes most biological membranes. The presence of discrete lipid domains in natural membranes is thought to have a profound impact on their biological function, as exemplified by cells ${ }^{13}$. A specific case of lateral organization is represented by lipid rafts, defined as micro and/or nano-domains, enriched in lipids such as cholesterol, sphingomyelin, saturated glycerophospholipids and glycosphingolipids: these lipids segregate in the so-called liquid-ordered phase $\left(L_{0}\right)$, which is immiscible with the surrounding liquid-crystalline (disordered, $L_{d}$ ) phase ${ }^{14}$. This phase heterogeneity induces a thickness mismatch between neighboring domains and the consequent, ergonically unfavorable, exposure of hydrocarbon regions to water, which results in an energetic cost, due to interfacial energy ${ }^{15}$. Rafts are thought to participate in the formation and targeting of nano-sized biogenic lipid vesicles (e.g. Extracellular Vesicles, EVs) ${ }^{16}$. They are also actively involved in multiple membrane processes, e.g. they act as structural platforms for organizing protein machinery ${ }^{17}$, they can preferentially associate with specific membrane proteins ${ }^{18}$ and represent centers for the assembly of signaling molecules. From a mechanical point of view, the presence of phase boundaries and, hence, bilayers thickness mismatches, generates deformations and increases membrane permeability ${ }^{19-21}$. All these structural perturbations 
promote the selective adsorption of materials on the membrane; indeed, as pointed out by Hamada et al. ${ }^{22}$, lateral heterogeneity, promoted by the presence of micro-sized lipid rafts, regulates the adsorption of nano/microparticles, with the larger ones preferring the Ldphases and the smaller ones being localized in the $L_{\circ}$ phases of cell-sized lipid vesicles. These selective NPs adsorption pathways are also present in the case of nano-sized lipid segregated domains and can be studied exploiting liposomes with tunable rafts size ${ }^{15}$. However, investigating the interaction of NPs with nanometric lipid rafts remains a major challenge, mainly hindered by the small size of the segregated domains, which makes standard optical techniques not suitable for the task. Recent studies demonstrated that gold nanoparticles (AuNPs) adsorb more strongly to phase-separated multicomponent lipid bilayers; in particular, they are believed to preferentially target the phase boundary regions, due to their intrinsic negative curvature ${ }^{23,24}$.

To the best of our knowledge, this behavior has only been investigated by computational studies $^{23}$ and experiments involving Quartz Crystal Microbalance $(\mathrm{QCM})^{24}$, which provide important but indirect evidences. In summary, the preferential adsorption of AuNPs along the boundaries of nano sized lipid domains has never been directly observed.

To fill this gap, we exploit Atomic Force Microscopy (AFM), to directly visualize the preferential adsorption of AuNPs on the phase boundaries of multicomponent SLBs, presenting both an Ld and an Lophase and previously characterized by Neutron Reflectivity (NR). The $L_{d}$ phase is made of 1,2-dioleoyl-sn-glycero-3-phosphocholine (DOPC) with two unsaturated hydrocarbon chains that hinder molecular packing, while the Lo phase is composed of 1,2-dipalmitoyl-sn-glycero-3-phosphocholine (DSPC) lipids and cholesterol molecules which occupy the free volume between the lipid acyl chains ${ }^{13,25}$. The quantitative localization and morphometry of AuNPs adsorbed on the SLB reveal important information regarding their interaction with the lipid matrix. The study corroborates the already theorized 
differential NPs-lipid interaction taking place at the phase boundaries of lipid rafts. The presented results could help the development of future NPs-based applications that involve their adsorption on membranes characterized by nanoscale phase segregations.

\section{MATERIALS AND METHODS}

\section{Materials}

Tetrachloroauric (III) acid ( $\geq 99.9 \%$ ), trisodium citrate dihydrate ( $\geq 99.9 \%$ ), methanol (99.8\%), $\mathrm{CHCl}_{3}(\geq 99.9 \%), \mathrm{NaCl}(\geq 99.5 \%)$ and $\mathrm{CaCl}_{2}(99.999 \%)$ were provided by SigmaAldrich (St. Louis, MO). The same for 1,2-dioleoyl-sn-glycero-3-phosphocholine (DOPC) ( $\geq$ 98.0\%), cholesterol ( $\geq 99.5 \%)$ and 1,2-distearoyl-sn-glycero-3-phosphocholine (DSPC) ( $\geq$ 98.0\%). All chemicals were used as received. Milli-Q grade water was used in all preparations.

\section{AuNP preparation}

Anionic gold nanospheres of $16 \mathrm{~nm}$ in size were synthesized according to the TurkevichFrens method 26,27 . Briefly, $20 \mathrm{ml}$ of a $1 \mathrm{mM} \mathrm{HAuCl}_{4}$ aqueous solution were brought to boiling temperature under constant and vigorous magnetic stirring. $2 \mathrm{ml}$ of $1 \%$ citric acid solution were then added and the solution was further boiled for 20 minutes, until it acquired a deep red color. The nanoparticles solution dispersion was then slowly cooled down to room temperature.

\section{Vesicle preparation and SLB formation for Neutron Reflectivity measurements}

\section{Vesicle preparation}

The proper amount of a DOPC/DSPC/cholesterol mixture (39/39/22 mol\%) was dissolved in chloroform and a lipid film was obtained by evaporating the solvent under a stream of nitrogen and overnight vacuum drying. The film was then swollen and suspended in warm 
$\left(50^{\circ} \mathrm{C}\right) 100 \mathrm{mM} \mathrm{NaCl}$ water solution by vigorous vortex mixing, in order to obtain a final $0.5 \mathrm{mg} / \mathrm{ml}$ lipid concentration. The resultant multilamellar vesicles (MLVs) were tip sonicated with a Digital Sonifier Model 450 (Branson), provided with a Horn Tip (diameter 25.4 mm), in an intermittent-pulse mode (5 seconds), with a power of $400 \mathrm{~W}$ (amplitude 50\%), for 15 minutes to obtain a homogeneous dispersion of unilamellar vesicles (ULVs).

\section{Surface cleaning procedure}

DOPC/DSPC/cholesterol single lipid bilayers were formed on $50 \times 80 \times 15 \mathrm{~mm}^{3}$ Silicon mirrors (Andrea Holm GmbH, Tann, Germany; roughness $\leq 5 \AA$ ). Substrates were preliminary rinsed in either ultrapure water and ethanol, in order to remove organic residues. After that, they were bath sonicated treated for 30 minutes in ethanol with a Bandelin DL 102 3L bath sonicator, followed by other 30 minutes in ultrapure water (Millipore Simplicity UV). The surfaces were then cleaned with a Novascan PSD-UV8 UV/ozone plasma for 30 min and rinsed in ultrapure water. Finally, they were dried with nitrogen gas and stored in ultrapure water, ready for the deposition.

\section{Vesicle fusion and SLB formation}

$\mathrm{CaCl}_{2}$ was added to the vesicle dispersion, reaching a final concentration of $10 \mathrm{mM}$, just before the injection in the NR measuring cell. This was performed in order to promote their adhesion to the support and their subsequent disruption. Vesicles were left incubating for 30 minutes; then, the saline buffer was switched to $\mathrm{D}_{2} \mathrm{O}$ to promote the vesicle disruption and SLB formation. The use of $\mathrm{D}_{2} \mathrm{O}$ instead of ultrapure water ensures a better resolution of the lipid structures for the NR measurements.

\section{Vesicle preparation and SLB formation for AFM measurements}

\section{Vesicle preparation}


The proper amount of a DOPC/DSPC/cholesterol mixture (39/39/22 mol\%) was dissolved in chloroform and a lipid film was obtained by evaporating the solvent under a stream of nitrogen and overnight vacuum drying. The film was then swollen and suspended in warm $\left(50^{\circ} \mathrm{C}\right)$ ultrapure water solution by vigorous vortex mixing, in order to obtain a final $0.5 \mathrm{mg} / \mathrm{ml}$ lipid concentration. The resultant multilamellar vesicles in water were subjected to 10 freezethaw cycles and extruded 10 times through two stacked polycarbonate membranes with 100 $\mathrm{nm}$ pore size at room temperature, to obtain unilamellar vesicles with narrow and reproducible size distribution. The filtration was performed with the Extruder (Lipex Biomembranes, Vancouver (Canada)) through Nuclepore membranes.

\section{Surface cleaning procedure}

All reagents were purchased from Sigma-Aldrich Inc (www.sigmaaldrich.com). DOPC/DSPC/Chol supported lipid bilayers were formed on microscopy borosilicate glass coverslips (Menzel Gläser). Glass slides were first immersed in a 3:1 mixture of $96 \% \mathrm{H}_{2} \mathrm{SO}_{4}$ and $50 \%$ aqueous $\mathrm{H}_{2} \mathrm{O}_{2}$ ('oxidizing piranha') solution for $2 \mathrm{~h}$ in order to remove any organic residue present on their surface. Then, the slides were cleaned in a sonicator bath (Elmasonic Elma $\mathrm{S} 30 \mathrm{H}$ ) for 30 minutes in acetone, followed by 30 minutes in isopropanol and 30 minutes in ultrapure water (Millipore Simplicity UV). Glass slides were then cleaned with air plasma for 15 minutes (Air plasma cleaner PELCO easiGlow) and incubated in ultrapure water for 10 minutes in order to maximize the number of reactive silanols present on the surface. Finally, they were dried with nitrogen gas and stick to a magnetic disk, ready for the lipid solution deposition.

\section{Vesicle fusion and SLB formation}

A $100 \mu \mathrm{l}$ droplet of buffer solution was firstly spotted on the $\mathrm{SiO}_{2}$ slide. The buffer solution consisted of $\mathrm{CaCl}_{2} 200 \mathrm{mM}$ diluted 1:10 in $\mathrm{KCl} 100 \mathrm{mM}$. A $10 \mu \mathrm{l}$ droplet containing the lipid mixture was then added to the buffer droplet and left incubating at room temperature for 30 
minutes in order to promote the vesicle adsorption on the surface. After that, the droplet was removed and replaced by a $100 \mu \mathrm{l}$ droplet of ultrapure water which was then left incubating for other 15 minutes. AuNPs deposition on the SLB was obtained by adding $5 \mu$ l of a $7.8 \mathrm{nM}$ AuNPs dispersion to the ultrapure water droplet and leaving it to incubate for 10 minutes. After the system equilibrated, the large droplet was gently removed and the slide was inserted in the AFM fluid cell for the measurements.

\section{Neutron Reflectivity measurements}

NR measurements were conducted at the REFSANS Horizontal TOF reflectometer of the Helmholtz-Zentrum Geesthacht located at the Heinz Maier-Leibnitz Zentrum in Garching, Germany ${ }^{28,29}$. Neutrons in the wavelength range $3.0-21.0 \AA$ were used to carry out the measurements. Two incident angles, namely $0.60^{\circ}$ and $3.00^{\circ}$, allowed collecting data in the range $0.007 \leq \mathrm{Q} / \AA^{-1} \leq 0.22$. The arrival times and positions of scattered neutrons were detected on a Denex 2D $500 \times 700 \mathrm{~mm}^{2}$ multiwire ${ }^{3} \mathrm{He}$ detector (pixel size $2.1 \times 2.9 \mathrm{~mm}^{2}$, efficiency $80 \%$ at $7 \AA$, gamma sensitivity $<10^{-6}$ ) positioned at $4.5 \mathrm{~m}$ from the sample. The detector was installed in a liftable vacuum tube in order to reach exit angles up to $5.2^{\circ}$ at the maximum elongation. In order to receive sufficient statistics, a counting time of about 4 hours for the measurement was chosen. The software MOTOFIT ${ }^{30}$ was employed for the analysis of the NR curve. Details on data analysis are reported in the SI.

\section{AFM measurements}

\section{AFM setup}

All AFM experiments were performed at room temperature on a Bruker Multimode 8 (equipped with Nanoscope $\mathrm{V}$ electronics, a sealed fluid cell and a type JV piezoelectric scanner) using Bruker SNL-A probes (triangular cantilever, nominal tip curvature radius 2$12 \mathrm{~nm}$, nominal elastic constant $0.35 \mathrm{~N} / \mathrm{m}$ ) calibrated with the thermal noise method ${ }^{31}$. The 
AFM fluid cell was filled with a saline buffer solution, consisting of $\mathrm{KCl} 100 \mathrm{mM}$, which has the main effect of reducing the Debye length that characterizes the Electrical Double Layer (EDL) interaction region between AFM tip and SLB ${ }^{32}$. In this way, better image resolution can be achieved.

\section{AFM Imaging}

Imaging was performed in PeakForce mode. In order to minimize deformations or rupture events induced by the scanning probe, the applied force setpoint was kept under $200 \mathrm{pN}$ range. Feedback gain was set on values high enough to obtain optimal image quality but low enough to prevent the introduction of noise signals that would otherwise interfere with the resolution of the different lipid domains (lipid phases have a height difference of $\sim 1 \mathrm{~nm}$ ). The average height value of all bare substrate zones was taken as the baseline zero height reference. Image background subtraction was performed using Gwyddion 2.53.16 ${ }^{33}$.

\section{RESULTS AND DISCUSSION}

\section{Formation of Supported Lipid Bilayers containing lipid rafts}

The formation of a continuous planar bilayer (DOPC/DSPC/cholesterol (39/39/22 mol\%)), covering the vast majority of the supporting surface, was achieved through vesicle fusion and characterized by NR. Briefly, as described in the Materials and Methods section, liposomes in a saline buffer were additioned with a low amount of $\mathrm{CaCl}_{2}$, injected within the measurement chamber and left adsorbing on the support (a clean Si crystal). The presence of $\mathrm{Ca}^{2+}$ ions in solution promotes the crowding of vesicles on the surface by reducing the repulsive interactions between liposomes with surface charge. As reported by Richter et al. ${ }^{9}$, when a critical vesicle coverage is reached, the stress on the vesicles becomes sufficient to induce their rupture; in our case the phenomenon was also favored by the additional osmotic shock, coming from the replacement of the saline buffer with ultrapure water. The edges of the newly-formed SLB are energetically unfavorable and cause the rupture of other surface- 
bioRxiv preprint doi: https://doi org/10.1101/2020.05.03.075101. this version posted May 4.2020 . The copyright holder for this preprint (which was not certified by peer review) is the author/funder, who has granted bioRxiv a license to display the preprint in perpetuity. It is made available under aCC-BY-NC-ND 4.0 International license.

bound vesicles. If the density of adsorbed vesicles is sufficiently high, these cascade phenomena can lead to the complete surface coverage.

Neutron Reflectivity (NR) was applied to probe the effective formation of the multicomponent SLB and its structure along the normal to the SLB plane. Figure 1a shows a representative NR profile measured for the SLB in $\mathrm{D}_{2} \mathrm{O}$ (green circles), together with the fitting curve (red continuous line). The curve was analyzed with MOTOFIT and, consistently with the literature ${ }^{34-36}$, it was possible to model the profile of the SLB as a stack of five layers (see scheme in Figure 1b): the silicon oxide layer, a layer of solvent $\left(\mathrm{D}_{2} \mathrm{O}\right)$, a layer for the polar headgroups in contact with the support (inner heads), a layer for the lipid chains (chains) and, finally, a layer for the polar headgroups in contact with the solvent (outer heads). Each layer is characterized by a defined contrast (the scattering length density, SLD), thickness (d), roughness $(\rho)$ and hydration (solvent \%). The curve fitting results are reported in Table 1. The overall thickness of the bilayer is $\sim 5 \mathrm{~nm}$ (given by the sum of the thickness values related to the inner and outer heads, plus the lipid chains). The negligible hydration $(0.1 \%)$ of the lipid chains layer indicates that the surface was almost completely covered by a homogeneous lipid bilayer. The analysis of the experimental data allowed reconstructing the entire profile of the SLB along the normal to the surface (see Figure 1b).

\section{$d(\AA) \quad \rho(\AA) \quad$ SLD $\left(10^{-6} \AA^{-2}\right) \quad$ solvent $\%$}

\begin{tabular}{l|cccc} 
Inner heads & $5 \pm 2$ & $2 \pm 1$ & 1.87 & $5 \pm 1$ \\
Lipid Chains & $38 \pm 3$ & $1 \pm 1$ & -0.18 & $0.1 \pm 0.1$ \\
Outer Heads & $6 \pm 2$ & $2 \pm 1$ & 1.87 & $16 \pm 4$
\end{tabular}

Table 1 Curve fitting results of NR data, obtained with MOTOFIT. The reported fitting parameters are referred to the three layers composing the bilayer (Inner Heads, referred to the layer of polar headgroups in contact with the support, Lipid Chains, referred to the hydrophobic region of the SLB, Outer Heads, referred to the layer of polar headgroups in contact with the solvent (i.e., $\left.D_{2} O\right)$ ). For each layer four parameters are reported: $d(\AA)$, the thickness of the layer; $\rho(\AA)$ roughness of the layer; SLD $\left(10^{-6} \AA^{-2}\right)$ scattering length density of the layer; Solvent \% $D_{2} \mathrm{O}$ penetration in each layer. 
While NR provides information on the average structure with respect to the bilayer normal, AFM can be used to resolve in detail the in-plane rafts morphology $37-40$. The SLB was formed on functionalized borosilicate glass coverslips, by injecting the liposomes (this time suspended in ultrapure water) in the buffer solution where they experienced an osmotic imbalance across the membrane, decreasing their pressurization (please refer to Materials and Methods for the details). As a result, following the adhesion to the substrate, liposomes will deform adopting more oblate shapes ${ }^{41}$, increasing the area occupied by each vesicle and favoring the previously described vesicle fusion mechanism. As shown in Figure 1c, consistently with NR data the surface is almost completely covered by a lipid bilayer, which presents nanometric domains of different heights, with the brighter areas corresponding to thicker membrane regions and the darker ones to thinner SLB portions. Accordingly, the height distribution of Figure 1d confirms the presence of two distinct lipid phases, with height values of $h_{d}=3.7 \mathrm{~nm}$ and $h_{o}=4.7 \mathrm{~nm}$, in good agreement with the results obtained by Heberle et al. on the same vesicle preparation ${ }^{15}$. This thickness mismatch can be ascribed to the coexistence of two lipid phases of different composition, dictating variations in the membrane's height ${ }^{42-44:}$ in particular, membrane thickness was found to increase with length or degree of saturation of the lipid tails ${ }^{43,44}$. Here the thicker domains can be associated with the Lo phase, which is enriched with cholesterol and DSPC, i.e. a fully saturated long chain lipid. On the contrary, thinner regions correspond to the $L_{d}$ lipid phase mainly composed of DOPC, which is characterized by a shorter tail length and two chain unsaturated bonds. After having properly flattened the image, by the application of a mask (see Figure S4), it is possible to determine the area fractions occupied by each of the two phases. Heberle et al. ${ }^{15}$ reported the area fraction corresponding to the $L_{d}$ phase for liposomes of the very same composition to be 0.52 ; our calculations on SLBs are in line with those findings, giving a $L_{d}$ area fraction of 0.50 . Results also suggest that the SLB formation did not significantly modify the amount of $L_{d}$ and $L_{o}$ lipids, originally present in the unfused 
vesicles and that the lipid phase behavior is not affected by the presence of the solid support. The presented results corroborate the essential role of AFM in providing comprehensive morphological details on structure of rafted membranes. In the following paragraph, we extend the existing literature on AFM-based rafts characterizations ${ }^{37-40}$, by studying the structure of lipid rafts following their interaction with AuNPs.

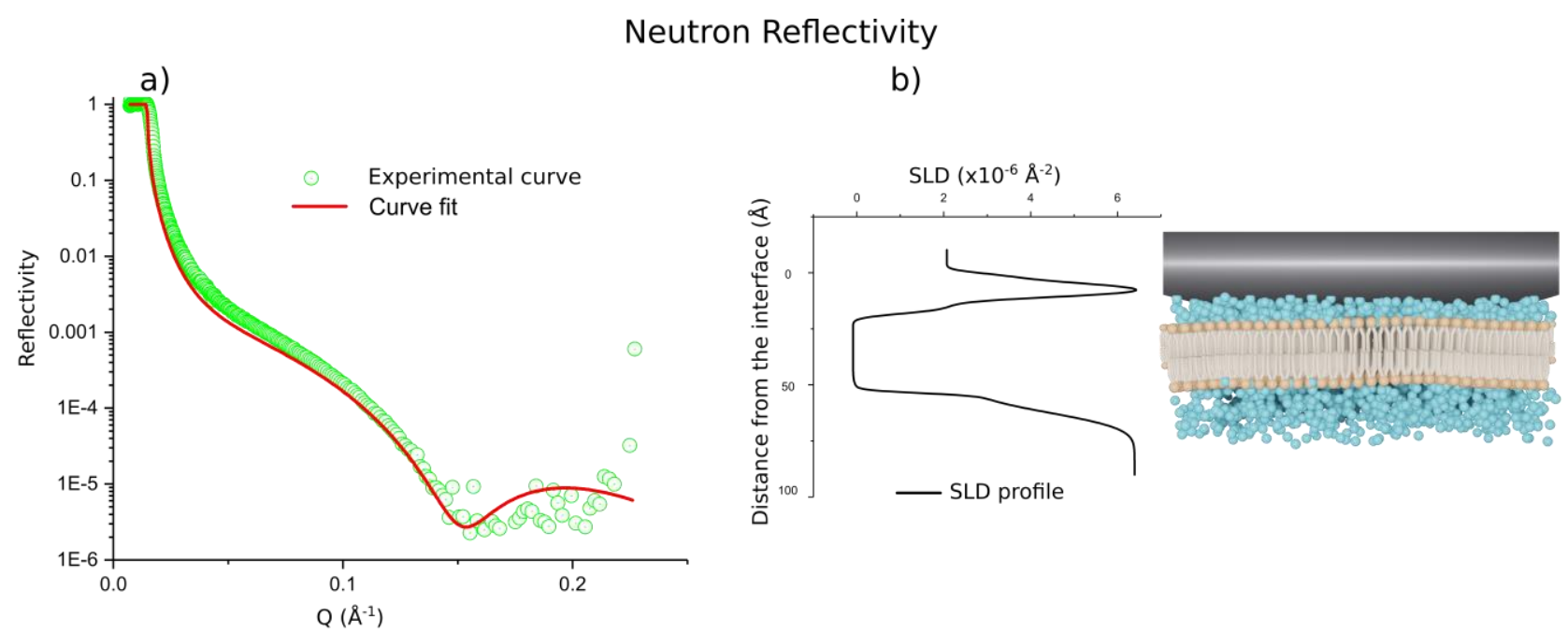

Atomic Force Microscopy

c)

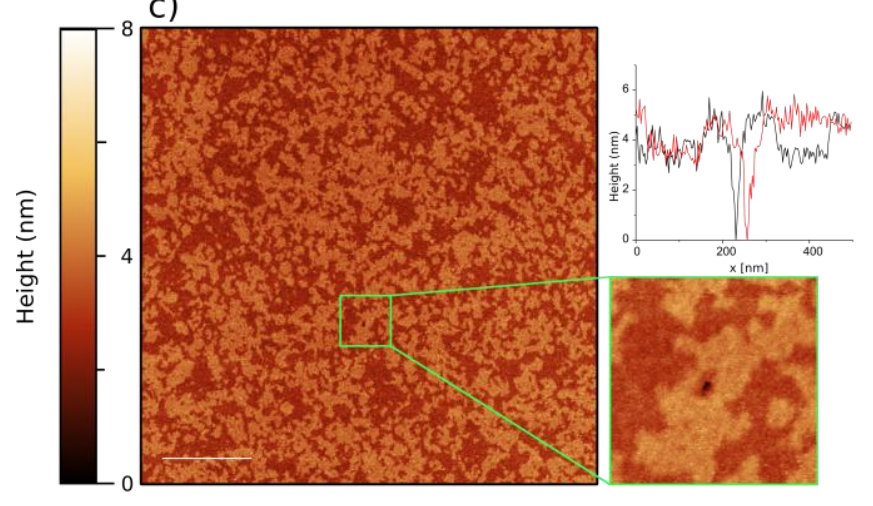

d)

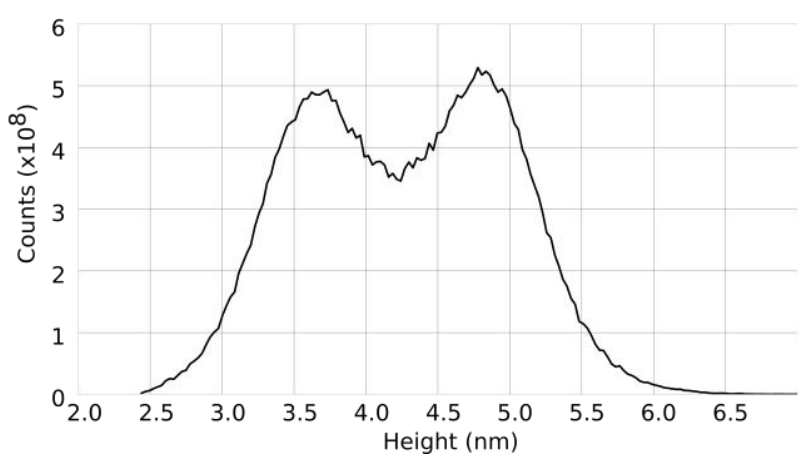

Figure 1 Characterization of the multicomponent SLB formed from DOPC/DSPC/cholesterol (39/39/22 mol\%) liposomes by vesicle fusion. a) Neutron Reflectivity profile (green circles) and best fit (continuous red line) corresponding to the SLB in $\mathrm{D}_{2} \mathrm{O}$; from the fitting analysis the average bilayer thickness is $-5 \mathrm{~nm}$. b) Scattering length density (SLD) profile, describing variations of the SLD along the direction perpendicular to the bilayer. c) Representative AFM topography of the SLB. The bilayer uniformly covers the surface, displaying both the $\mathrm{L}_{o}$ (brighter thicker regions) and $\mathrm{L}_{d}$ phases (darker thinner regions) as segregated domains. The reported scalebar is $1 \mu \mathrm{m}$. The $500 \times 500 \mathrm{~nm}$ micrograph (bottom inset) displays the small hole in the bilayer that allowed flattening the image with respect to the $\mathrm{SiO}_{2}$ surface. Two perpendicular height profiles were traced, horizontally and vertically, across the whole image (top inset); the profiles confirm the presence of the two distinct lipid phases covering the surface. d) Height distribution obtained from the AFM image; the two distinct peaks, centered at $h_{d}=3.7 \mathrm{~nm}$ and $h_{o}=4.7 \mathrm{~nm}$, describe the different heights that characterize the $L_{d}$ and $L_{o}$ phase, respectively. 


\section{Interaction of AuNPs with lipid rafts: localization of AuNPs at the boundaries}

In order to investigate the interaction of $16 \mathrm{~nm}$ citrated AuNPs (please refer to Materials and Methods for AuNP synthesis and to SI for AuNPs characterization details) with the lipid rafts present in the SLB, $5 \mu \mathrm{l}$ of the NPs dispersion were injected in the ultrapure water buffer. Different literature reports connect the presence of phase segregation within the lipid bilayer to the selective adsorption of NPs along the domains boundaries ${ }^{23,24}$; however, a direct proof of this interaction is still missing to date. AFM represents one of the few techniques that could provide the sufficient resolution to simultaneously resolve the height difference between the two lipid phases $(\sim 1 \mathrm{~nm})$ and the morphology of AuNPs. Despite the high resolution provided by $\mathrm{AFM}$, the measurement remains challenging, as the spontaneous attachment of AuNPs to the probe (Figure S5) can often lead to imaging artifacts. In order to overcome this problem, the AFM fluid cell was filled with the same saline buffer used for SLB formation and the force SetPoint was kept on very low values (lower than $\sim 200 \mathrm{pN}$ ). The use of the saline buffer as imaging solution should compensate the tip-sample electrical double-layer repulsion ${ }^{32}$ and limit the attachment of the NPs to the probe. In order to identify the portions of lipid bilayer characterized by the presence of AuNPs, images of $5 \times 5 \mu \mathrm{m}$ regions were initially acquired. Figure 2 a shows a representative AFM topography of the SLB following the NPs injection. The bigger spherical objects represent vesicles that still have to fuse within the bilayer, while the smaller ones are the AuNPs, which seem to be homogeneously distributed above the SLB. 
a)

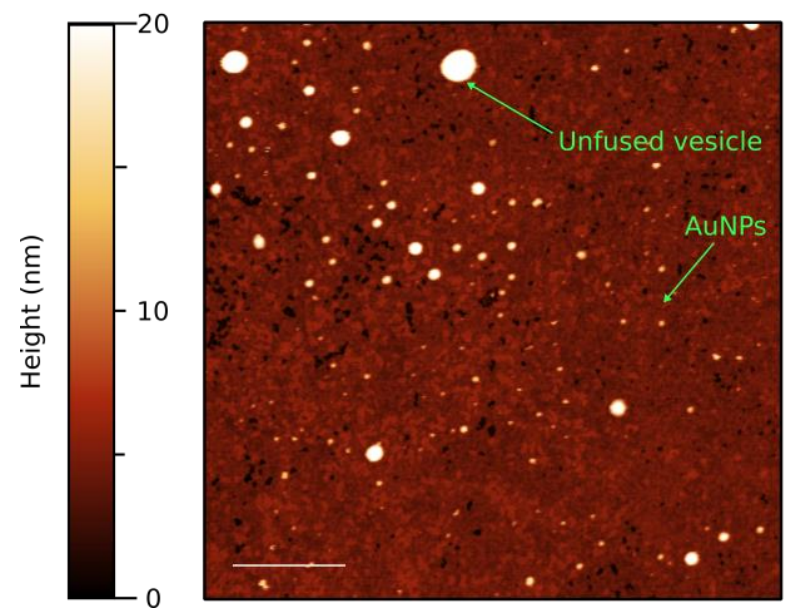

b)

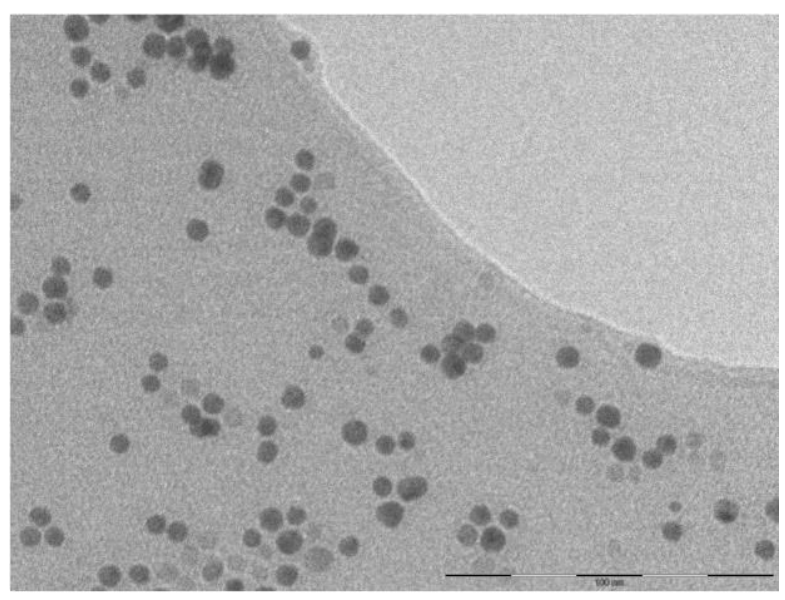

Figure 2 a) Representative AFM topography of the SLB following the injection of AuNPs. Lipid rafts are still visible as differently shaded areas. The larger and heterogeneous spherical objects represent unfused vesicles while the smaller ones are the AuNPs that have been homogeneously adsorbed on the lipid bilayer. Scalebar is $1 \mu \mathrm{m}$. b) Transmission Electron Microscopy (TEM) image of the AuNPs that were used in the experiments, scalebar is $100 \mathrm{~nm}$ (please refer to the SI for details regarding TEM characterization).

From a simple AFM topography, small lipid vesicles can be confused with AuNPs or AuNPs clusters; this could introduce statistical noise to the localization and morphometrical analysis. We recently developed an AFM-based nanomechanical characterization able to discriminate lipid vesicles from objects with the same morphology but different mechanical behavior $^{41}$. This method evaluates the deformation that lipid vesicles undergo once adsorbed on a surface, by calculating their contact angle $(\alpha)$. Through the measurement of $\alpha$ and by assuming that the surface area of the vesicles is preserved upon adsorption, it is also possible to evaluate the diameter that characterizes the unperturbed vesicles in solution (called Diameter in solution). As described in Figure 3, lipid vesicles are characterized by a narrow distribution of contact angles over a wide range of sizes (Diameter in solution), while AuNPs present a narrow size distribution and higher contact angle values. This enables the easy singling out of the AuNPs and their exclusive inclusion in the next analysis. 


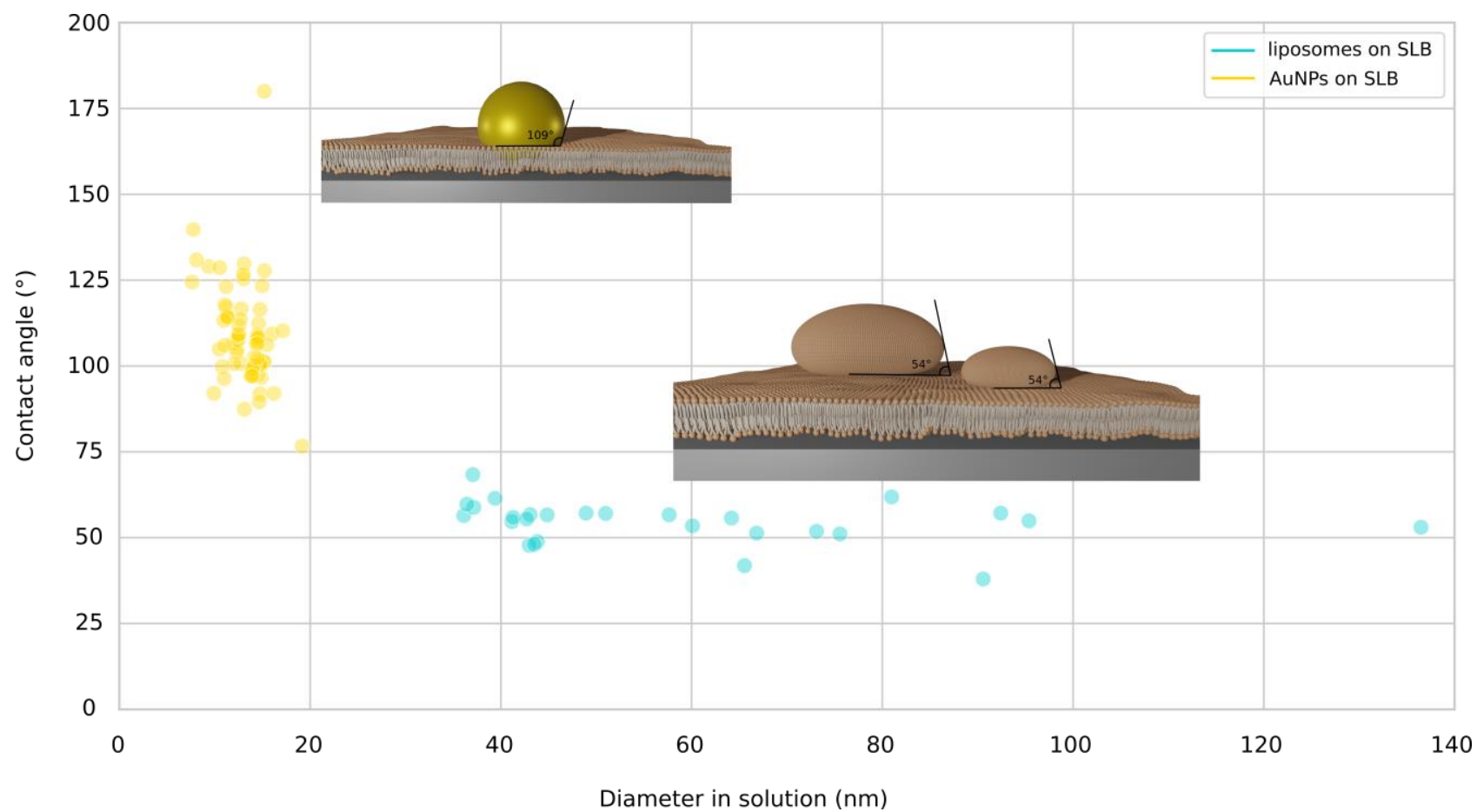

Figure 3 Plot representing the distributions of contact angle vs solution diameter of either vesicles (blue circles) and AuNPs (yellow circles). Vesicles data have been obtained from the liposomes present in Figure 2a while the AuNPs data come from micrographs like the ones reported in Figure 4a. Even though adsorbed on the SLB, liposomes show their nanomechanical fingerprint: a narrow contact angle distribution over a wide range of sizes. Their average contact angle is $\sim 54^{\circ}$ hence describing highly deformed shapes, possibly due to the SLB formation procedure. AuNPs display narrow distributions for both their size and contact angle, with average values of $14 \mathrm{~nm}$ and $109^{\circ}$, respectively.

In order to precisely determine whether the NPs targeted specific locations on the lipid matrix, the size of the scanned region was further reduced. In Figure 4a, representative images, with sizes of $\sim 600 \times 600 \mathrm{~nm}$, illustrating the SLB decorated by AuNPs have been reported. The micrographs of Figure 4a constitute the direct proof of the AuNPs selective adsorption along the segregated phase boundaries. 
a)
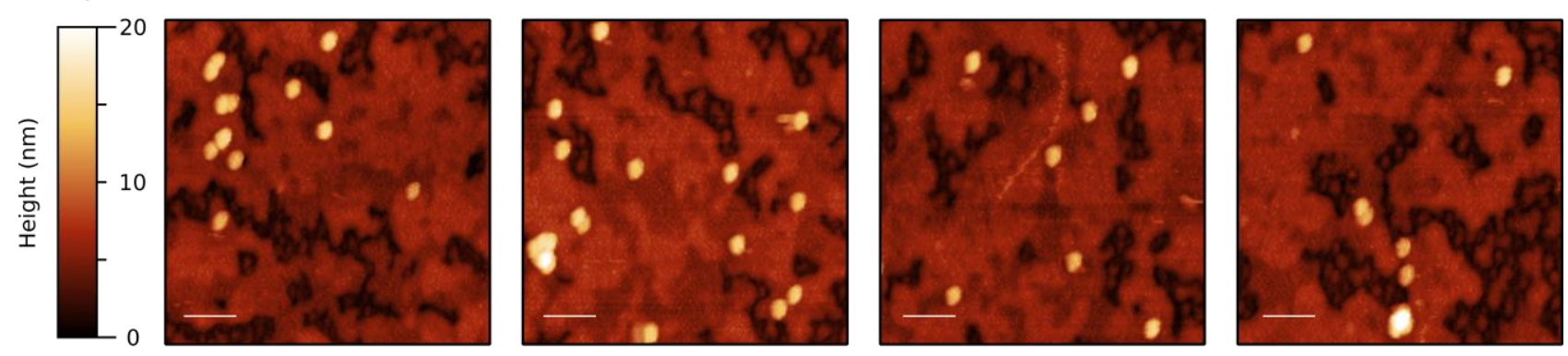

b)
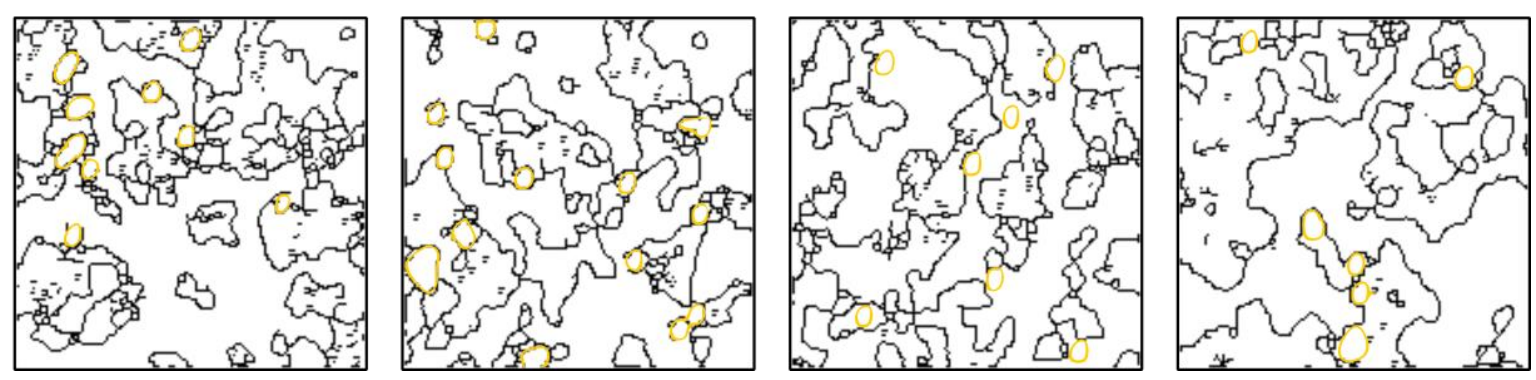

Figure 4 a) Representative AFM micrographs that clearly display the selective adsorption of AuNPs along the boundaries of the lipid rafts (brighter regions of the SLB that correspond to the Lo lipid phase). From the images it is also possible to distinguish between isolated and clustered NPs. All scalebars are $100 \mathrm{~nm}$. b) Contour images obtained from the micrographs. Black lines represent the rafts edges while gold circles define the contours of the AuNPs. The gold NPs edges are always in contact with at least one of the lines describing the lipid segregated phase boundaries.

In the free image processing software Gwyddion 2.53.16, the sequential application of different masks allowed mapping the edges of either the lipid rafts and NPs shown in Figure $4 a$ and, hence, obtaining a clearer indication of their relative positions. In Figure $4 b$ the contour images of NPs and rafts have been superimposed with different colors, to highlight that AuNPs preferentially targeted the boundaries of the two lipid phases; indeed, the lines describing their shapes are always in contact with the edges of the lipid rafts. These results further corroborate the hypothesis that phase boundaries represent energetically favorable niches for lipid-NPs interactions. As previously discussed elsewhere ${ }^{23}$, NPs adsorption induces bilayer bending, which entails an energy penalty that increases the free energy associated with the overall process. This energy penalty is almost completely reduced along the phase boundaries, where the local negative curvature of the membrane, caused by the thickness mismatch between the two lipid phases minimizes the free energy associated with the NPs adsorption ${ }^{23}$. 


\section{Inclusion of AuNPs within the lipid bilayer}

AuNPs have a diameter of $16 \mathrm{~nm}$ (refer to SI for details), which is close to the average height measured with AFM imaging $(14 \pm 2 \mathrm{~nm})$. This suggests that after adsorbing on the SLB, AuNPs probably penetrate the bilayer and reach the $\mathrm{SiO}_{2}$ surface. This result further extends the characterization of NPs-lipid interaction and corroborates our vision of rafts' boundaries as regions of increased permeability ${ }^{19-21}$, where the membrane can easily wrap around the adsorbed NPs. Recent findings (Montis et al., JCIS, accepted on $31^{\text {st }}$ March 2020) confirm these results, suggesting that free-standing lipid bilayers can bend around the AuNPs surface, guided by citrate-lipid ligand exchange at the interface. All the above hypotheses are confirmed by the evaluation of the AuNPs contact angle with respect to the SLB. As suggested by Vinelli et al. ${ }^{45}$, the contact angle of a perfectly spherical, non-deformable (under the considered forces) object should be $180^{\circ}$, while we measured a substantially lower value. These apparent discrepancies can be rationalized by a careful morphological analysis, as detailed below.

The size of AuNPs is comparable with the tip radius, hence the effect of tip convolution should be taken into account. This was performed by assuming the NPs as perfectly spherical and non-deformable objects with heights that coincide with their actual diameters. This is a reasonable assumption given that, during an AFM measurement, the error along the vertical direction is negligible compared to the ones in the scanning plane. As a consequence, all the measured radii were then corrected by $6 \mathrm{~nm}$ (corresponding to half the difference between the average NPs height and diameter measured by AFM). The NPs average contact angle, calculated with respect to the SLB and by using the corrected radii, gave a value of $109^{\circ}$, which is in very good agreement with the result that can be obtained from a simple geometrical model (Figure 5), featuring a $14 \mathrm{~nm}$ spherical and undeformable 
NP immersed in $a \sim 5 \mathrm{~nm}$ lipid bilayer. For that case, a would be equal to $107^{\circ}$; this last result confirms that AuNPs penetrated the lipid bilayer and reached the underlying substrate.

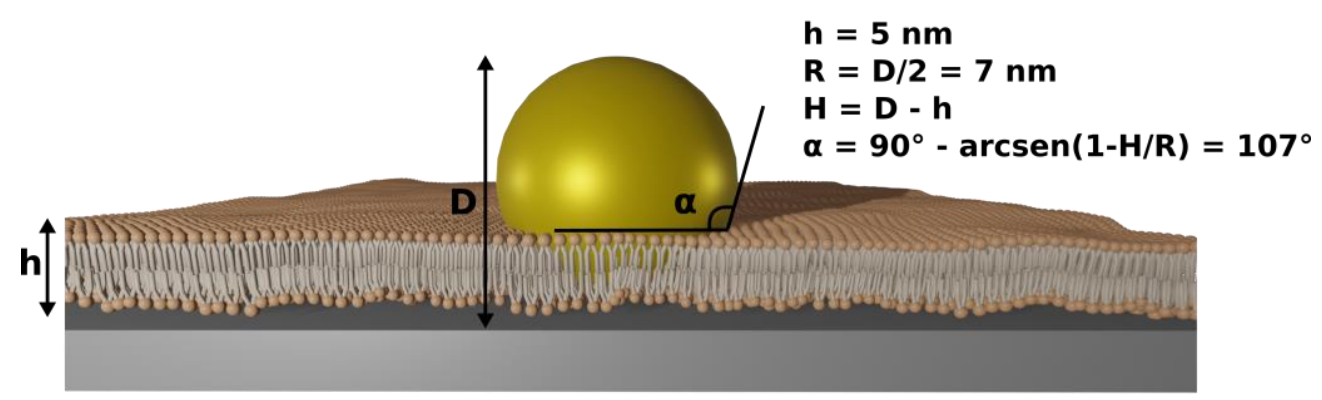

Figure 5 Schematic representation of the configuration used to evaluate, from a conceptual point of view, the contact angle that would characterize an AuNP with a diameter of $14 \mathrm{~nm}$, adsorbed on a rigid flat surface and surrounded by a $\sim 5 \mathrm{~nm}$ lipid bilayer.

\section{CONCLUSION}

The presence of lipid rafts within the cell membrane has been linked with multiple important biological functions, like the formation and targeting of lipid nano-vesicles. The thickness mismatch that originates between the different immiscible segregated domains is thought to generate mechanical stresses, that enhance the membrane permeability along these regions. We herein exploited Atomic Force Microscopy to investigate the preferential adsorption of AuNPs along the phase boundaries of SLBs, generated from DOPC/DSPC/cholesterol (39/39/22 mol\%) liposomes. Different works in the literature suggested a selective adsorption of AuNPs along the boundaries of lipid segregated domains, but a direct observation of this phenomenon is still missing to date. AFM allowed us to probe the existence of nanometric lipid rafts on the newly formed SLB and to spot the presence of NPs along their edges, hence providing a direct proof of this preferential adsorption pathway. In addition, we provided useful details about the experimental procedures that could significantly improve the reliability of AFM imaging; indeed, one of the major challenges hindering this type of measurements is the frequent tip contamination, caused by the attachment of the NPs to the AFM probe. We showed that the use of a saline 
buffer as imaging solution within the AFM fluid cell leads to optimal image quality and strongly reduces tip contamination events. Then, through the application of an AFM-based morphometric nanomechanical characterization, it was also possible to further investigate the reorganization of the lipid bilayer, as a consequence of the AuNPs adsorption. We found out that the lipid matrix wrapped around the NPs, allowing them to penetrate within the hydrophobic region until reaching the rigid $\mathrm{SiO}_{2}$ surface of the slides. The theoretical calculation of the morphological parameters describing this phenomenon is in perfect agreement with the experimental results and further corroborates our interpretation. Further studies will focus on extending this characterization to membranes with varying compositions and employing NPs of different core and/or size.

\section{AUTHOR INFORMATION}

Corresponding Author

*E-mail: andrea.ridolfi@ismn.cnr.it

\section{Author Contributions}

The manuscript was written through contributions of all authors. All authors have given approval to the final version of the manuscript.

Notes The authors declare no competing financial interests.

\section{ACKNOWLEDGMENTS}

This work was also supported by the Consorzio Sistemi a Grande Interfase (CSGI) through the evFOUNDRY project, Horizon 2020-Future and emerging technologies (H2020FETOPEN), ID: 801367. We thank the SPM@ISMN research facility for support in the AFM experiments. Maier-Leibnitz Zentrum is acknowledged for provision of beam-time. 


\section{SUPPLEMENTARY INFORMATION}

\section{AuNPs characterization}

\section{TRANSMISSION ELECTRON MICROSCOPY}

Trasmission electron microscopy (TEM) images were acquired with a STEM CM12 Philips electron microscope equipped with an OLYMPUS Megaview G2 camera, at CeME (CNR Florence Research Area, Via Madonna del Piano, 10 - 50019 Sesto Fiorentino). Drops of citrated AuNP, diluted ten times, were placed on 200 mesh carbon-coated copper grids with a diameter of $3 \mathrm{~mm}$ and a thickness of $50 \mu \mathrm{m}$ (Agar Scientific) and dried at room temperature. Then, samples were analyzed at an accelerating voltage of $100 \mathrm{keV}$.

\section{SMALL ANGLE X-RAY SCATTERING (SAXS)}

SAXS measurements were carried out on a S3-MICRO SAXS/WAXS instrument (HECUS $\mathrm{GmbH}$, Graz, Austria) which consists of a GeniX microfocus X-ray sealed Cu Ka source (Xenocs, Grenoble, France) of $50 \mathrm{~W}$ power which provides a detector focused X-ray beam with $\lambda=0.1542 \mathrm{~nm} \mathrm{Cu} \mathrm{Ka}$ line. The instrument is equipped with two one-dimensional (1D) position sensitive detectors (HECUS 1D-PSD-50 M system), each detector is $50 \mathrm{~mm}$ long (spatial resolution $54 \mu \mathrm{m} /$ channel, 1024 channels) and cover the SAXS q-range $(0.003<\mathrm{q}$ $\left.<0.6 \AA^{-1}\right)$. The temperature was controlled by means of a Peltier TCCS-3 Hecus. The analysis of SAXS curves was carried out using lgor Pro ${ }^{46}$. SAXS measurements on AuNP aqueous dispersions, was carried out in sealed glass capillaries of $1.5 \mathrm{~mm}$ diameter. To analyze AuNPs profiles, we chose a model function with a spherical form factor and a Schulz size distribution ${ }^{47}$, which calculates the scattering for a polydisperse population of spheres 
with uniform scattering length density. The distribution of radii (Schulz distribution) is given by the following equation:

$$
(R)=(z+1)^{z+1} x^{z} \frac{\exp [-(z+1) x]}{R_{\text {avg }} \Gamma(z+1)}
$$

where $R_{\text {avg }}$ is the mean radius, $x=R / R_{a v g}$ and $z$ is related to the polydispersity of the dispersion. The form factor is normalized by the average particle volume, using the $3^{\text {rd }}$ moment of R:

$$
\langle V\rangle=\frac{4 \pi}{3}\left\langle R^{+3}\right\rangle=\frac{4 \pi}{3}\langle R\rangle^{3} \frac{(z+3)(z+2)}{(z+1)^{2}}
$$

The scattering intensity is:

$$
I(q)=\left(\frac{4 \pi}{3}\right)^{2} N_{0} \Delta \rho^{2} \int_{0}^{\infty} f(R) R^{6} F^{2}(q R) d R
$$

where $N_{0}$ is the total number of particles per unit volume, $F(R)$ is the scattering amplitude for a sphere and $\Delta \rho$ is the difference in the scattering length density between the AuNP and the solvent.

The structural parameters of citrated gold nanoparticles were evaluated from the SAXS profile of Figure S1 according to the above model.

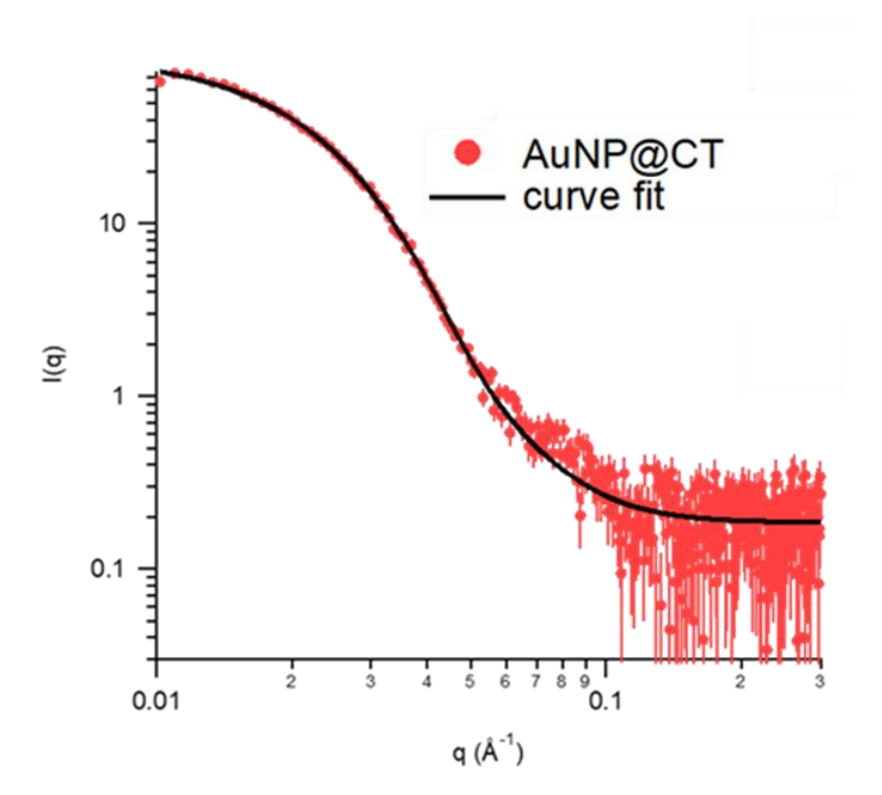


Figure S1 Experimental SAXS profile (markers) obtained for citrated AuNPs and curve fit (solid black line) according to the Schulz spheres model from the NIST package SANS Utilities. The size and polydispersity obtained from the fitting procedure are $13 \mathrm{~nm}$ and 0.3 , respectively.

\section{UV-VIS SPECTROSCOPY}

UV-Vis spectra were measured with a JASCO UV-Vis spectrophotometer.

The size of citrate gold nanoparticles was further evaluated from UV-Vis Spectroscopy by the following equation ${ }^{48}$ :

$$
d=\exp \left(B_{1} \frac{A_{s p r}}{A_{450}}-B_{2}\right)
$$

with d diameter of gold nanoparticles, Aspr absorbance at the surface plasmon resonance peak, $A_{450}$ absorbance at the wavelength of $450 \mathrm{~nm}$ and $B_{1}$ and $B_{2}$ dimensionless parameters, taken as 3 and 2.2, respectively. The obtained diameter value is $16 \mathrm{~nm}$.

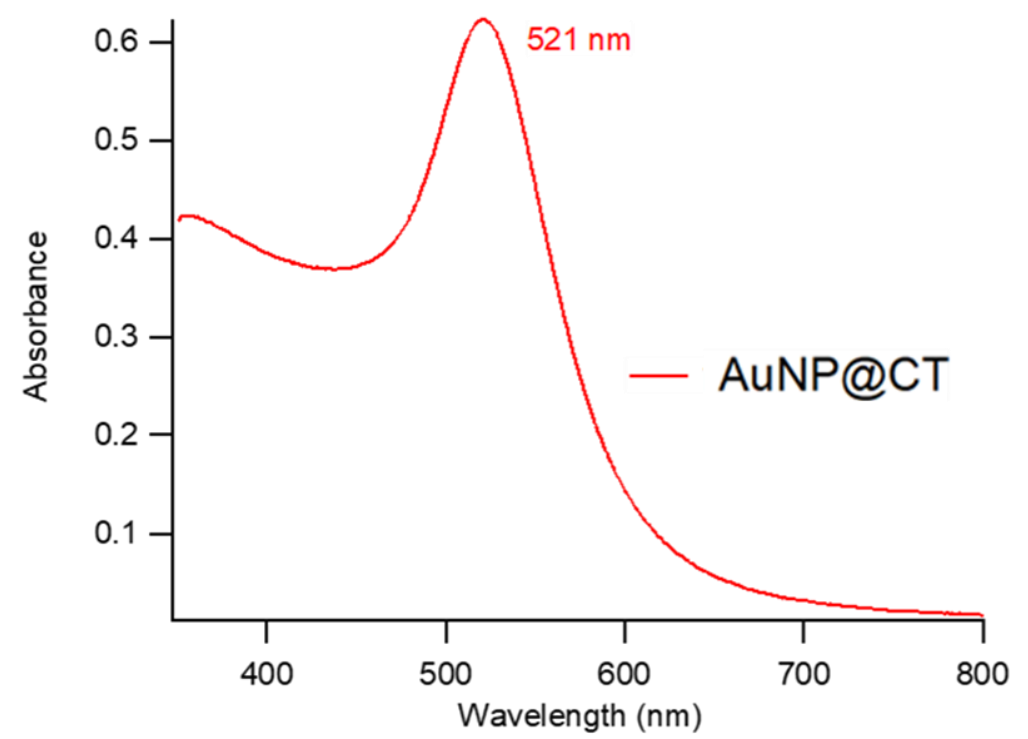

Figure S2 UV-Vis absorption spectra of citrated AuNP dispersion (after 1:5 dilution in water). The plasmon absorption peak is located at $521 \mathrm{~nm}$.

The concentration of citrated gold nanoparticles was determined via UV-Vis spectrometry, using the Lambert-Beer law $(E(\lambda)=\varepsilon(\lambda)$ lc $)$ and considering the extinction values $\varepsilon(\lambda)$ at the 
LSPR maximum, i.e. $\lambda=521 \mathrm{~nm}$. The extinction coefficient $\varepsilon(\lambda)$ was determined by the following equation ${ }^{49}$ :

$$
\ln (\varepsilon)=k \ln (d)+a
$$

with $\mathrm{d}$ core diameter of nanoparticles, and $\mathrm{k}$ and a dimensionless parameter $(\mathrm{k}=3.32111$ and $\mathrm{a}=10.80505)$. The arithmetic mean of the size, obtained by both the optical and the scattering analyses, leads to an $\varepsilon(\lambda)$ value of $4.8 \cdot 10^{8} \mathrm{M}^{-1} \mathrm{~cm}^{-1}$. Consequently, the final concentration of citrated AuNP is $\sim 7.8 \cdot 10^{-9} \mathrm{M}$.

\section{ATOMIC FORCE MICROSCOPY}

Preliminary AFM images of AuNPs adsorbed on bare mica substrates were performed in order to test the effect of using a saline buffer as imaging solution, to prevent the attachment of NPs to the AFM tip (please refer to the Materials and Methods section for details regarding the imaging setup and parameters).
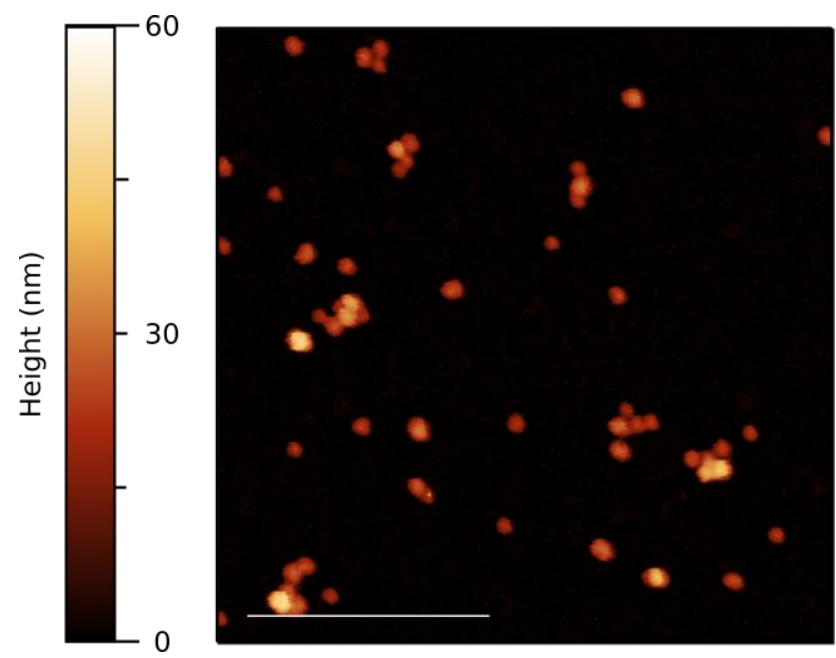

Figure S3 AFM topography representing AuNPs either isolated or clustered, adsorbed on a bare mica substrate. As reported in the Materials and Methods section, AFM imaging was performed using a saline buffer as imaging solution, in order to obtain better image quality and avoid tip contamination due to the attachment of the NPs to the probe. As can be seen from the image, isolated AuNPs get faithfully described by nearly perfect spherical shapes with an average contact angle close to $180^{\circ}$. Scalebar is $400 \mathrm{~nm}$.

\section{Neutron Reflectivity measurements}


The software MOTOFIT was employed for the analysis of the NR curves. A five-layer model was employed to analyze the reflectivity profiles of neat SLBs, with scattering length density values calculated for each layer: a bulk subphase of $\operatorname{Si}\left(\operatorname{SLD}=2.07^{*} 10^{-6} \AA^{-2}\right)$, a superficial layer of $\mathrm{SiO}_{2}\left(\mathrm{SLD}=3.47^{*} 10^{-6} \AA^{-2}\right)$; a second layer of $\mathrm{D}_{2} \mathrm{O}\left(\mathrm{SLD}=6.393 * 10^{-6} \AA^{-2}\right)$; a third layer composed of the polar headgroups of the SLB of the inner leaflet (SLD $=1.87^{*} 10^{-6} \AA^{-}$ 2); a fourth layer composed of the bilayer's lipid chains (SLD $=-0.18 * 10^{-6} \AA^{-2}$ ); a fifth layer composed of the polar headgroups of the outer bilayer's leaflet (SLD $=1.87^{*} 10^{-6} \AA^{-2}$ ); a bulk superphase of solvent $\left(\mathrm{D}_{2} \mathrm{O}, \mathrm{SLD}=6.393{ }^{*} 10^{-6} \AA^{-2}\right)$. The scattering length density values for the polar headgroups and lipid chains were estimated by taking into account the chemical compositions and the submolecular fragment volumes of phosphatidylcholines as determined by Armen et al. through molecular dynamic simulations ${ }^{50}$ (see table S1).

Table S1. Chemical formula, molecular volumes and corresponding scattering length densities of species relevant to this study

$\begin{array}{cccc}\text { Molecule } & \text { Chem. Formula } & \text { Volume }\left(\AA^{3}\right) & \operatorname{SLD}\left(10^{-6} \AA^{-2}\right) \\ \text { PC headgroups } & \mathrm{C}_{10} \mathrm{H}_{18} \mathrm{NO}_{8} \mathrm{P} & 321.9 & 1.866 \\ \text { DSPC chains } & \mathrm{C}_{34} \mathrm{H}_{70} & 1004.6 & -0.357 \\ \text { DOPC chains } & \mathrm{C}_{34} \mathrm{H}_{66} & 982.2 & -0.212 \\ \text { cholesterol } & \mathrm{C}_{27} \mathrm{H}_{46} \mathrm{O} & 630 & 0.210\end{array}$


bioRxiv preprint doi: https://doi.org/10.1101/2020.05.03 075101 t this version posted May 4, 2020. The copyright holder for this preprint (which was not certified by peer review) is the author/funder, who has granted bioRxiv a license to display the preprint in perpetuity. It is made available under aCC-BY-NC-ND 4.0 International license.

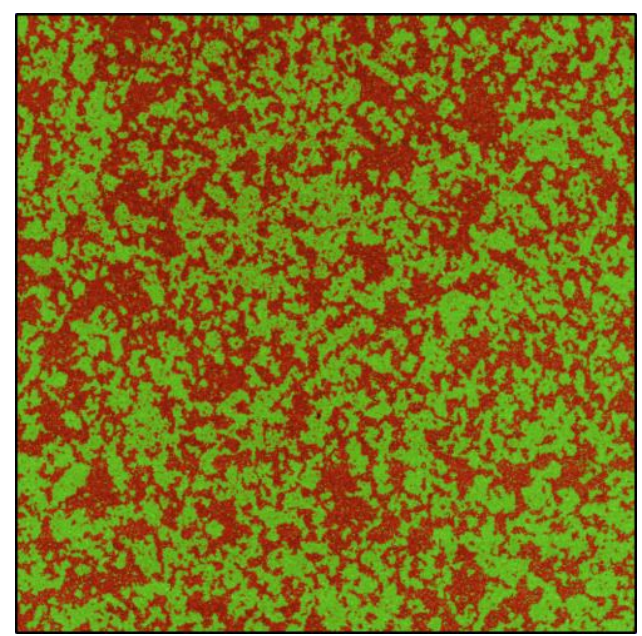

Figure S4 AFM topography of the SLB obtained from DOPC/DSPC/Chol (39/39/22 \%w/w) liposomes. Using Gwyddion 2.53.16 it was possible to apply a mask to selectively cover the Lo phase (characterized by higher height values) and estimate the area fraction of each phase. The area fractions of the $L_{o}$ and $L_{d}$ phases are approximately 0.50 , confirming the results obtained by Heberle et al. ${ }^{15}$ on the very same vesicles preparation.

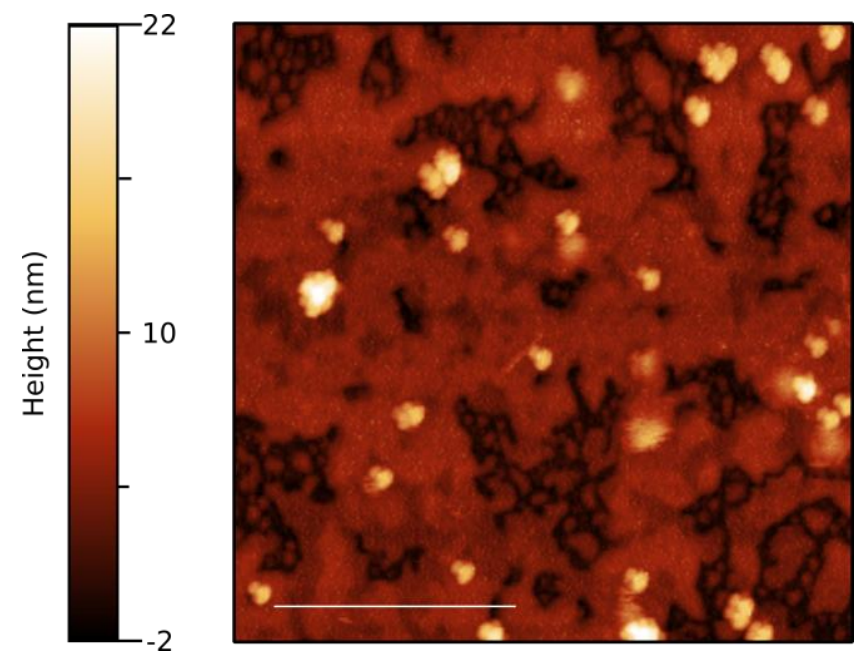

Figure S5 AFM topography showing the effects of tip contamination on the imaging of AuNPs adsorbed on the lipid bilayer. While the SLB gets correctly imaged, all the NPs appear in "clusters" characterized by similar shapes (same protrusions in all the directions). This is a clear indicator that the AFM probe has been contaminated by the attachment of one or multiple NPs which lead to the generation of imaging artifacts. Scalebar is $400 \mathrm{~nm}$. 


\section{BIBLIOGRAPHY}

1. Henriksen-Lacey, M., Carregal-Romero, S., and Liz-Marzán, L.M. (2017). Current challenges toward in vitro cellular validation of inorganic nanoparticles. Bioconjug. Chem. 28, 212-221.

2. Nel, A.E., Mädler, L., Velegol, D., Xia, T., Hoek, E.M.V., Somasundaran, P., Klaessig, F., Castranova, V., and Thompson, M. (2009). Understanding biophysicochemical interactions at the nano-bio interface. Nat. Mater. 8, 543-557.

3. Beddoes, C.M., Case, C.P., and Briscoe, W.H. (2015). Understanding nanoparticle cellular entry: A physicochemical perspective. Adv. Colloid Interface Sci. 218, 48-68.

4. Mendozza, M., Caselli, L., Salvatore, A., Montis, C., and Berti, D. (2019). Nanoparticles and organized lipid assemblies: From interaction to design of hybrid soft devices. Soft Matter 15, 8951-8970.

5. Zendrini, A., Paolini, L., Busatto, S., Radeghieri, A., Romano, M., Wauben, M.H.M., van Herwijnen, M.J.C., Nejsum, P., Borup, A., Ridolfi, A., et al. (2020). Augmented COlorimetric NANoplasmonic (CONAN) Method for Grading Purity and Determine Concentration of EV Microliter Volume Solutions. Front. Bioeng. Biotechnol. 7,.

6. Simonelli, F., Bochicchio, D., Ferrando, R., and Rossi, G. (2015). Monolayer-Protected Anionic Au Nanoparticles Walk into Lipid Membranes Step by Step. J. Phys. Chem. Lett. 6, 3175-3179.

7. Gkeka, P., Sarkisov, L., and Angelikopoulos, P. (2013). Homogeneous hydrophobichydrophilic surface patterns enhance permeation of nanoparticles through lipid membranes. J. Phys. Chem. Lett. 4, 1907-1912.

8. Su, J., Esmaeilzadeh, H., Zhang, F., Yu, Q., Cernigliaro, G., Xu, J., and Sun, H. (2018). An ultrasensitive micropillar-based quartz crystal microbalance device for real-time measurement of protein immobilization and protein-protein interaction. Biosens. Bioelectron. $99,325-331$. 
9. Richter, R.P., Bérat, R., and Brisson, A.R. (2006). Formation of solid-supported lipid bilayers: An integrated view. Langmuir 22, 3497-3505.

10. Hardy, G.J., Nayak, R., and Zauscher, S. (2013). Model cell membranes: Techniques to form complex biomimetic supported lipid bilayers via vesicle fusion. Curr. Opin. Colloid Interface Sci. 18, 448-458.

11. Nikoleli, G.P., Nikolelis, D.P., Siontorou, C.G., Nikolelis, M.T., and Karapetis, S. (2018). The application of lipid membranes in biosensing. Membranes (Basel). 8,.

12. Worsfold, O., Voelcker, N.H., and Nishiya, T. (2006). Biosensing using lipid bilayers suspended on porous silicon. Langmuir 22, 7078-7083.

13. Sezgin, E., Levental, I., Mayor, S., and Eggeling, C. (2017). The mystery of membrane organization: Composition, regulation and roles of lipid rafts. Nat. Rev. Mol. Cell Biol. 18, 361-374.

14. Koynova, R., and Tenchov, B. (2013). Transitions between lamellar and nonlamellar phases in membrane lipids and their physiological roles. OA Biochem. 1,.

15. Heberle, F.A., Petruzielo, R.S., Pan, J., Drazba, P., Kučerka, N., Standaert, R.F., Feigenson, G.W., and Katsaras, J. (2013). Bilayer thickness mismatch controls domain size in model membranes. J. Am. Chem. Soc. 135, 6853-6859.

16. Busatto, S., Zendrini, A., Radeghieri, A., Paolini, L., Romano, M., Presta, M., and Bergese, P. (2020). The nanostructured secretome. Biomater. Sci. 8, 39-63.

17. Lingwood, D., and Simons, K. (2010). Lipid rafts as a membrane-organizing principle. Science (80-. ). 327, 46-50.

18. Simons, K., and lkonen, E. (1997). Functional rafts in cell membranes. Nature 387, 569572.

19. Rawicz, W., Smith, B.A., Mclntosh, T.J., Simon, S.A., and Evans, E. (2008). Elasticity, strength, and water permeability of bilayers that contain raft microdomain-forming lipids. Biophys. J. 94, 4725-4736. 
20. Sheikh, K.H., and Jarvis, S.P. (2011). Crystalline hydration structure at the membranefluid interface of model lipid rafts indicates a highly reactive boundary region. J. Am. Chem. Soc. $133,18296-18303$.

21. Kuzmin, P.I., Akimov, S.A., Chizmadzhev, Y.A., Zimmerberg, J., and Cohen, F.S. (2005). Line tension and interaction energies of membrane rafts calculated from lipid splay and tilt. Biophys. J. 88, 1120-1133.

22. Hamada, T., Morita, M., Miyakawa, M., Sugimoto, R., Hatanaka, A., Vestergaard, M.C., and Takagi, M. (2012). Size-dependent partitioning of nano/microparticles mediated by membrane lateral heterogeneity. J. Am. Chem. Soc. 134, 13990-13996.

23. Sheavly, J.K., Pedersen, J.A., and Van Lehn, R.C. (2019). Curvature-driven adsorption of cationic nanoparticles to phase boundaries in multicomponent lipid bilayers. Nanoscale $11,2767-2778$.

24. Melby, E.S., Mensch, A.C., Lohse, S.E., Hu, D., Orr, G., Murphy, C.J., Hamers, R.J., and Pedersen, J.A. (2016). Formation of supported lipid bilayers containing phasesegregated domains and their interaction with gold nanoparticles. Environ. Sci. Nano 3, 4555.

25. Toppozini, L., Meinhardt, S., Armstrong, C.L., Yamani, Z., Kučerka, N., Schmid, F., and Rheinstädter, M.C. (2014). Structure of cholesterol in lipid rafts. Phys. Rev. Lett. 113,. 26. Turkevich, J., Stevenson, P.C., and Hillier, J. (1951). A study of the nucleation and growth processes in the synthesis of colloidal gold. Discuss. Faraday Soc. 11, 55-75.

27. FRENS, G. (1973). Controlled Nucleation for the Regulation of the Particle Size in Monodisperse Gold Suspensions. Nat. Phys. Sci. 241, 20-22.

28. Moulin, J.-F., and Haese, M. (2015). REFSANS: Reflectometer and evanescent wave small angle neutron spectrometer. J. Large-Scale Res. Facil. JLSRF 1,.

29. Kampmann, R., Haese-Seiller, M., Kudryashov, V., Nickel, B., Daniel, C., Fenzl, W., Schreyer, A., Sackmann, E., and Rädler, J. (2006). Horizontal ToF-neutron reflectometer 
REFSANS at FRM-II Munich/Germany: First tests and status. Phys. B Condens. Matter 385-386, 1161-1163.

30. Nelson, A. (2006). Co-refinement of multiple-contrast neutron/X-ray reflectivity data using MOTOFIT. J. Appl. Crystallogr. 39, 273-276.

31. Hutter, J.L., and Bechhoefer, J. (1993). Calibration of atomic-force microscope tips. Rev. Sci. Instrum. 64, 1868-1873.

32. Müller, D.J., Fotiadis, D., Scheuring, S., Müller, S.A., and Engel, A. (1999). Electrostatically balanced subnanometer imaging of biological specimens by atomic force microscope. Biophys. J. 76, 1101-1111.

33. Nečas, D., and Klapetek, P. (2012). Gwyddion: An open-source software for SPM data analysis. Cent. Eur. J. Phys. 10, 181-188.

34. Montis, C., Gerelli, Y., Fragneto, G., Nylander, T., Baglioni, P., and Berti, D. (2016). Nucleolipid bilayers: A quartz crystal microbalance and neutron reflectometry study. Colloids Surfaces B Biointerfaces 137, 203-213.

35. Luchini, A., Nzulumike, A.N.O., Lind, T.K., Nylander, T., Barker, R., Arleth, L., Mortensen, K., and Cárdenas, M. (2019). Towards biomimics of cell membranes: Structural effect of phosphatidylinositol triphosphate (PIP 3 ) on a lipid bilayer. Colloids Surfaces B Biointerfaces 173, 202-209.

36. Montis, C., Salvatore, A., Valle, F., Paolini, L., Carlà, F., Bergese, P., and Berti, D. (2020). Biogenic supported lipid bilayers as a tool to investigate nano-bio interfaces. J. Colloid Interface Sci. 570, 340-349.

37. Mingeot-Leclercq, M.P., Deleu, M., Brasseur, R., and Dufrêne, Y.F. (2008). Atomic force microscopy of supported lipid bilayers. Nat. Protoc. 3, 1654-1659.

38. Yuan, C., Furlong, J., Burgos, P., and Johnston, L.J. (2002). The size of lipid rafts: An atomic force microscopy study of ganglioside GM1 domains in sphingomyelin/DOPC/cholesterol membranes. Biophys. J. 82, 2526-2535. 
39. Cai, M., Zhao, W., Shang, X., Jiang, J., Ji, H., Tang, Z., and Wang, H. (2012). Direct evidence of lipid rafts by in situ atomic force microscopy. Small 8, 1243-1250.

40. Milhiet, P.E., Vié, V., Giocondi, M.C., and Le Grimellec, C. (2001). AFM characterization of model rafts in supported bilayers. Single Mol. 2, 109-112.

41. Ridolfi, A., Brucale, M., Montis, C., Caselli, L., Paolini, L., Borup, A., Boysen, A.T., Loria, F., Herwijnen, M.J.C. van, Kleinjan, M., et al. (2019). AFM-based High-Throughput Nanomechanical Screening of Single Extracellular Vesicles. BioRxiv.

42. Bleecker, J. V., Cox, P.A., Foster, R.N., Litz, J.P., Blosser, M.C., Castner, D.G., and Keller, S.L. (2016). Thickness Mismatch of Coexisting Liquid Phases in Noncanonical Lipid Bilayers. J. Phys. Chem. B 120, 2761-2770.

43. Lewis, B.A., and Engelman, D.M. (1983). Lipid bilayer thickness varies linearly with acyl chain length in fluid phosphatidylcholine vesicles. J. Mol. Biol. 166, 211-217.

44. Petrache, H.I., Dodd, S.W., and Brown, M.F. (2000). Area per lipid and acyl length distributions in fluid phosphatidylcholines determined by $2 \mathrm{H}$ NMR spectroscopy. Biophys. J. 79, 3172-3192.

45. Vinelli, A., Primiceri, E., Brucale, M., Zuccheri, G., Rinaldi, R., and Samorì, B. (2008). Sample preparation for the quick sizing of metal nanoparticles by atomic force microscopy. Microsc. Res. Tech. 71, 870-879.

46. Kline, S.R. (2006). Reduction and analysis of SANS and USANS data using IGOR Pro. J. Appl. Crystallogr. 39, 895-900.

47. Kotlarchyk, M., and Chen, S.H. (1983). Analysis of small angle neutron scattering spectra from polydisperse interacting colloids. J. Chem. Phys. 79, 2461-2469.

48. Haiss, W., Thanh, N.T.K., Aveyard, J., and Fernig, D.G. (2007). Determination of size and concentration of gold nanoparticles from UV-Vis spectra. Anal. Chem. 79, 4215-4221. 49. Liu, X., Atwater, M., Wang, J., and Huo, Q. (2007). Extinction coefficient of gold nanoparticles with different sizes and different capping ligands. Colloids Surfaces B 
bioRxiv preprint doi: https://doi.org/10.1101/2020.05.03.075101; this version posted May 4, 2020. The copyright holder for this preprint (which was not certified by peer review) is the author/funder, who has granted bioRxiv a license to display the preprint in perpetuity. It is made available under aCC-BY-NC-ND 4.0 International license.

Biointerfaces 58, 3-7.

50. Armen, R.S., Uitto, O.D., and Feller, S.E. (1998). Phospholipid component volumes:

Determination and application to bilayer structure calculations. Biophys. J. 75, 734-744. 